Abstract ID: 17

\title{
Development of the malay digit triplet test via headphones and telephones as a national hearing screening tool in Malaysia
}

\author{
Saiful Adli Jamaluddin ${ }^{a}$ | Greg A. O'beirne ${ }^{b}$ | Nurlin Ali Hanafiah ${ }^{a}$ | Sarah Rahmat ${ }^{a}$ \\ anternational Islamic University Malaysia \\ ${ }^{b}$ University of Canterbury, New Zealand
}

Introduction: This study aimed to develop a self-administered hearing screening test using digit triplets in Malay (MDTT) to be used as a national hearing screening tool via internet and telephone landlines in Malaysia. Methods: Recordings of disyllabic Malay digits by a female Malay native speaker were used together with a speech shaped stationary noise (TSN). The test stimuli were normalized using responses from twenty normal hearing native Malay speakers. The data were used to produce eight equivalent test lists. An additional twenty normal hearing native Malay speakers were recruited to test the lists equivalency. Forty six listeners with varying levels of hearing were recruited to validate the tests. ROC analysis was done to identify the pass and refer cut-off limits. Results: No statistical differences were found between lists for both test conditions. The evaluation in fixed SNRs resulted in a mean speech reception threshold (SRT) of $-11.3 \pm 0.34 \mathrm{~dB}$ SNR for headphone and $-10.24 \pm 0.1 \mathrm{~dB}$ SNR for telephone applications. A significant main effect of type of transducer was observed in both normal and hearing impaired groups. The cut off for refer, sensitivity and specificity for the tests using telephones are $(-5.8 \mathrm{~dB}, 80.8 \%, 95 \%)$ and headphones $(-9.8 \mathrm{~dB}, 80.8 \%, 100 \%)$. Conclusions: The MDTT was successfully developed and the normative values are comparable to other versions of the digit triplet tests. The MDTT showed high sensitivity and specificity and can be made available to any Malay speaking public via telephone and internet.

KEYWORDS: Digit Triplet, Hearing screening, Malay 